\section{Intraventricular Hemorrhage}

Elliot J. Roth

Department of Physical Medicine and Rehabilitation, Northwestern University, Feinberg School of Medicine, Chicago, IL, USA

\section{Definition}

An intraventricular hemorrhage is bleeding into the ventricles of the brain, where the cerebrospinal fluid normally circulates. It can be caused by trauma or spontaneously by hemorrhaging in stroke. It also is fairly common in premature infants of very low birth weight. It is found in up to one-third of all patients with moderate to severe traumatic brain injury. In trauma patients, it is usually associated with brain contusion or intracerebral hemorrhage and therefore often carries a poor prognosis. In both trauma and stroke, it can cause hydrocephalus and elevated intracranial pressure, which are often manifested by changes in cognitive functioning, drowsiness, and headache. These require immediate treatment.

\section{Cross-References}

> Intracerebral Hemorrhage

- Intracranial Hemorrhage

- Subarachnoid Hemorrhage

\section{References and Reading}

Findlay, J. M. (2000). Intraventricular hemorrhage. Neurosurgery Quarterly, 10, 182-195.

Naff, N. J. (1999). Intraventricular hemorrhage in adults. Current Treatment Options in Neurology, 1, 173-178. 